\title{
C-reactive protein is expressed in a cell-type specific manner in the murine thymus.
}

Shahan Mamoor, MS1

1Thomas Jefferson School of Law

San Diego, CA 92901

mamoorsk@tjsl.edu

C-reactive protein, or CRP, is an acute phase protein $(1,2)$ synthesized and released from the liver (3). CRP is transcriptionally induced during systemic inflammatory responses $(1,2)$. CRP expression in the thymus has previously been reported but in the context of promiscuous gene expression of self-antigen during negative selection $(4,5)$ or after ectopic expression $(6)$. Here, by comparing the transcriptomes of MTEC and CTEC from the thymuses of mice at 1,3 and 6 months using a published dataset (7), we found that CRP was among the genes whose expression changed most significantly between cTEC and mTEC at 3 months of murine life. CRP was expressed at significantly higher amounts in mTEC compared to cTEC. Thus, CRP, a molecule typically thought of as expressed by the liver and induced during systemic inflammatory responses, is expressed in a cell-type specific manner during mammalian development in the thymus.

Keywords: CRP, c-reactive protein, mTEC, cTEC, thymus, differential gene expression, systems biology of the immunologically active cells of the thymus. 


\section{Introduction}

The C-reactive protein is an innate immune signaling effector $(1,2)$ produced by the liver (3) during the acute phase of a systemic inflammatory response $(1,2,3)$. Its expression is used as a surrogate marker of inflammation in the clinic (8) as well as a correlate of risk of a cardiovascular event (9).

CRP expression in the thymus has been reported in the context of studies examine the role of AIRE in promiscuous transcriptional induction of peripheral tissue antigen (PTA) or tissue-restricted antigens (TRA) $(4,5)$. "Ectopic" CRP expression in mTEC has also been reported by studies using a transgene expressing human CRP in mice (6). Here, we found by analysis of a published dataset (7) that CRP was among the genes most differentially expressed in the thymus of wild-type mice at 3 months of age. Thus, expression of CRP, a protein produced in liver as a component of the systemic inflammatory response, is patterned in a cell-type specific manner in the thymus during mammalian development.

\section{Methods}

GSE56928 (7) was utilized for this differential gene expression analysis, performed using GEO2R. This published dataset was generated by Ki et al. using microarray (Affymetrix Mouse 4302.0 array) of cTEC, mTEChi and mTEClo, $n=2$ for each developmental time point ( 1 month, 3 month, and 6 months) (7). GSE102527 was also utilized for this analysis (10). Koh et al. used the gene (transcript) version of Affymetrix Mouse Gene 2.0 ST Array to generate the dataset used here. The Benjamini and Hochberg method of $p$-value adjustment was utilized for $p$-value calculation by GEO2R, and auto-detect option was used for log-transformation applied to global expression data. The NCBI generated category of platform annotation was used. When the expression of two groups was assessed to determined statistical significance, a two-tailed, unpaired T-test with Welch's correction was utilized. For statistical testing to determine the significance of difference between average RNA expression values across each developmental 
time point for three groups (cTEC, mTEChi and mTEClo), an ordinary one-way ANOVA with multiple comparisons (Tukey's multiple comparisons test) was utilized (PRISM 8.1.2) (227).

\section{Results}

$\underline{\mathrm{CRP}}$ is differentially expressed in the epithelium of the thymus

We used a public dataset to understand which genes are expressed most differently between medullary and cortical thymic epithelial cells of the thymus and across development of the mouse at months 1,3 , and 6 of development. We found that the C-reactive protein (CRP) was among the most differentially expressed genes in the thymus (Table 1).

At 3 months, CRP is one of the most differentially expressed genes in the TEC transcriptome when comparing the global gene expression profiles of mTEClo, mTEChi, and CTEC

Differential gene expression analyses can be performed in a number of different ways to answer different questions. First, we compared the transcriptomes of three cell types: cTEC, mTEChi $^{\text {hi }}$ and mTEClo at three time points: 1 month, 3 months, and 6 months (e.g., comparing cTEC, mTEChi and mTEClo at 1 month).

At each of these developmental time points, CRP was among the genes whose expression changed the most significantly with the transcriptomes of all three cell types considered. At 1 month, CRP was the 617th most differentially expressed gene out of 45101 transcripts between the three cell types (Table 1; $p=1.99 \mathrm{E}-06)$. At 6 months, CRP was the 977th most differentially expressed gene out of 45101 transcripts (Table $1 ; p=1.18 E-05)$. However, at 3 months, CRP was the 25th most differentially expressed gene out of 45101 transcripts (Table $1 ; p=3.74 E-09$ ).

At 3 months, CRP was expressed at significantly higher levels in mTEClo and in mTEChi than in cTEC. CRP was expressed at higher levels in mTEClo and mTEChi than in cTEC and this difference was also statistically significant (Figure 1B and Figure 1C, $p=0.0156$ and $p=0.0076$, respectively). CRP expression in $\mathrm{mTEClo}^{\mathrm{l}}$ was also higher than in mTEChi at 3 months (Figure $1 \mathrm{D} ; \mathrm{p}=0.0440)$. 
CRP is differentially expressed in a cell-type specific manner in the mTEClo of the thymus throughout murine development

Another way to understand differential gene expression in the TEC of the thymus using this dataset is to ask, by comparing two transcriptomes, what genes most significantly change in expression between these two specific cell types at certain developmental time points (e.g., comparing mTEClo and cTEC at 1 month, at 3 months, or at 6 months).

We found that CRP was one of the most differentially expressed genes in mTEClo across mammalian development in the thymus of the mouse when compared to the transcriptome of the cTEC (Table 1). At 1 month, CRP differential expression was ranked 180 out of 45101 transcripts (Table $1 ; p=1.43 E-05)$, at 3 months, CRP was the 2 nd most differentially expressed transcript out of 45101 transcripts (Table $1 ; \mathrm{p}=1.9 \mathrm{E}-08$ ); by 6 months, CRP was the most differentially expressed transcript out of all 450101 transcripts compared between mTEClo and cTEC (Table 1; $\mathrm{p}=1.36 \mathrm{E}-08)$.

At 1 month, 3 months, and at 6 months, the level of CRP expression was significantly higher levels in mTEClo than in cTEC (Figure 2). This was statistically significant at 1 month (Figure 2A; $p=0.0679$ ), at 3 months (Figure 2B; $p=0.0156$ ), and at 6 months (Figure 2C; $p=0.0005)$. The statistical significance of this difference increased from 3 to 1 months and from 6 to 3 months. Thus, statistically, the significance of the difference in CRP expression between mTEClo and cTEC increased through mammalian development.

We found that CRP was differentially expressed in the mTEClo of the thymus, was the gene whose expression changed the most, out of over 45,000 transcripts, between mTEClo and CTEC of the thymus, and it was expressed at significantly higher levels in the mTEClo than in cTEC.

\section{Expression of CRP does not change upon deletion of AIRE}

Many genes that are expressed by mTEC for negative selection are dependent on AIRE for promiscuous transcriptional activation. Thus, we probed a third dataset (10) to answer 
whether the expression of CRP in mTEC of the thymus required AIRE. This dataset possessed transcriptome data for wild-type mTEC and mTEC with a deletion in the AIRE gene.

In AIRE-deleted mTEC, the expression of CRP did not significantly change, as evidenced by the fact that the rank of differential expression for CRP when comparing AIREdeleted and AIRE-sufficient mTEC was 32294 out of 33732 total transcripts, and was not considered significantly differential (Table $1 ; p=0.966)$.

This indicates that AIRE does not control expression of CRP and supports the conclusion, in conjunction with the fact that mTEClo expressed just as much, and even more CRP than mTEChi, that CRP is not expressed in the mTEC of the thymus for purposes of negative selection.

\section{Discussion}

We found using a published dataset (7) of whole transcriptome analysis of the cortical and medullary epithelial cells of the thymus that isolated mTEC and cTEC from the thymus of wild-type mice at 1 month, 3 months, and 6 months of life that across murine development, that CRP is expressed in the thymus of mice, it is one of the genes whose expression changes the most out of the total transcriptome between mTEC and CTEC cell types of the thymus, and that CRP was expressed at significantly higher levels in mTEClo than in both cTEC and mTEChi.

CRP expression in the thymus has previously been reported in the context of promiscuous gene expression induced by AIRE. We found that upon deletion of AIRE (10), that the difference in CRP expression between AIRE-deleted and AIRE-sufficient mTEC was not significantly differential (Table 1; AIRE wt vs AIRE ko mTEC; $p=0.966$ ). In fact, greater than 95\% of the transcriptome changed in expression to greater degrees between AIRE knock-out and wild-type mTEC than gene expression of CRP did. Expression of CRP was significantly higher in mTEC expressing low levels of MHC-II than in mTEC expressing high levels of MHC-

II. Thus, we found endogenous CRP expression in the thymus and conclude that CRP expression in the thymus may serve some purpose aside from presentation of self-antigen for negative selection. 
We found stage- and cell type-specific patterning of CRP expression in the immunologically active cells of the thymus, a gene typically associated with induced expression in the liver during systemic inflammatory responses (1-3) with an emphasis on expression in the mTEClo cell type. CRP should be deleted specifically in the thymus of wildtype mice to assess the function of CRP in the medulla and cortex of the thymus (11-13). 


\section{References}

1. Hurlimann, J., Thorbecke, G.J. and Hochwald, G.M., 1966. The liver as the site of Creactive protein formation. The Journal of experimental medicine, 123(2), pp.365-378.

2. Black, S., Kushner, I. and Samols, D., 2004. C-reactive protein. Journal of Biological Chemistry, 279(47), pp.48487-48490.

3. Ciliberto, G., Arcone, R., Wagner, E.F. and Rüther, U., 1987. Inducible and tissue-specific expression of human C-reactive protein in transgenic mice. The EMBO journal, 6(13), pp. 4017-4022.

4. Derbinski, J., Schulte, A., Kyewski, B. and Klein, L., 2001. Promiscuous gene expression in medullary thymic epithelial cells mirrors the peripheral self. Nature immunology, 2(11), pp. 1032-1039.

5. Derbinski, J., Gäbler, J., Brors, B., Tierling, S., Jonnakuty, S., Hergenhahn, M., Peltonen, L., Walter, J. and Kyewski, B., 2005. Promiscuous gene expression in thymic epithelial cells is regulated at multiple levels. The Journal of experimental medicine, 202(1), pp.33-45.

6. Klein, L., Klein, T., Rüther, U. and Kyewski, B., 1998. CD4 T cell tolerance to human Creactive protein, an inducible serum protein, is mediated by medullary thymic epithelium. The Journal of experimental medicine, 188(1), pp.5-16.

7. Ki, S., Park, D., Selden, H.J., Seita, J., Chung, H., Kim, J., Iyer, V.R. and Ehrlich, L.I., 2014 Global transcriptional profiling reveals distinct functions of thymic stromal subsets and age-related changes during thymic involution. Cell reports, 9(1), pp.402-415.

8. Pepys, M.B. and Hirschfield, G.M., 2003. C-reactive protein: a critical update. The Journal of clinical investigation, 111(12), pp.1805-1812.

9. Rohde, L.E., Hennekens, C.H. and Ridker, P.M., 1999. Survey of C-reactive protein and cardiovascular risk factors in apparently healthy men. The American journal of cardiology, 84(9), pp.1018-1022.

10. Koh, A.S., Miller, E.L., Buenrostro, J.D., Moskowitz, D.M., Wang, J., Greenleaf, W.J., Chang, H.Y. and Crabtree, G.R., 2018. Rapid chromatin repression by Aire provides precise control of immune tolerance. Nature immunology, 19(2), pp.162-172.

11. Murata, S., Sasaki, K., Kishimoto, T., Niwa, S.I., Hayashi, H., Takahama, Y. and Tanaka, K., 2007. Regulation of CD8+ T cell development by thymus-specific proteasomes. Science, 316(5829), pp.1349-1353. 
12. Ohigashi, I., Zuklys, S., Sakata, M., Mayer, C.E., Zhanybekova, S., Murata, S., Tanaka, K., Holländer, G.A. and Takahama, Y., 2013. Aire-expressing thymic medullary epithelial cells originate from $\beta 5 t$-expressing progenitor cells. Proceedings of the National Academy of Sciences, 110(24), pp.9885-9890.

13. Ohigashi, I., Zuklys, S., Sakata, M., Mayer, C.E., Hamazaki, Y., Minato, N., Hollander, G.A. and Takahama, Y., 2015. Adult thymic medullary epithelium is maintained and regenerated by lineage-restricted cells rather than bipotent progenitors. Cell reports, 13(7), pp. 1432-1443. 


\begin{tabular}{|c|c|c|c|c|c|}
\hline cTEC vs mTEChi vs mTEClo & Rank & ID & p-value & $\mathbf{F}$ & \\
\hline 1 month & 617 & 1421946_at & 1.99E-06 & 109.689856 & \\
\hline 3 months & 25 & 1421946_at & 3.74E-09 & $3.68 \mathrm{E}+02$ & \\
\hline 6 months & 977 & 1421946_at & $1.18 \mathrm{E}-05$ & $8.14 \mathrm{E}+01$ & \\
\hline cTEC vs mTEChi & Rank & ID & p-value & $\mathbf{t}$ & B \\
\hline 1 month & 2281 & 1421946_at & 5.01E-05 & 9.0012181 & 2.402528 \\
\hline 3 months & 151 & 1421946_at & $3.64 \mathrm{E}-08$ & -21.7665971 & 9.689725 \\
\hline 6 months & 2278 & 1421946_at & 3.05E-04 & 7.30 & 0.670327 \\
\hline cTEC vs mTEClo & Rank & ID & p-value & $\mathbf{t}$ & B \\
\hline 1 month & 180 & 1421946_at & 1.43E-05 & 13.0588063 & 4.00126507 \\
\hline 3 months & 2 & 1421946_at & $1.9 \mathrm{E}-08$ & -23.8009474 & 9.13957 \\
\hline 6 months & 1 & 1421946_at & $1.36 \mathrm{E}-08$ & 37.840992 & 9.26724 \\
\hline mTEChi vs mTEClo & Rank & ID & p-value & $\mathbf{t}$ & B \\
\hline 1 month & 782 & 1421946_at & 7.93E-06 & $-1.08 E+01$ & 4.367439 \\
\hline 3 months & 4119 & 1421946_at & $6.08 \mathrm{E}-04$ & -5.8325835 & -0.317214 \\
\hline 6 months & 6578 & 1421946_at & $6.43 \mathrm{E}-03$ & -4.179069 & -2.61234 \\
\hline \multirow[t]{2}{*}{ mTEC vs AIRE ko mTEC } & Rank & ID & p-value & & \\
\hline & 32294 & 17219623 & $9.66 \mathrm{E}-01$ & & \\
\hline
\end{tabular}

Table 1: CRP is differentially expressed in the epithelium of the thymus

The rank of differential expression out of 45101 total transcripts detected and measured in the dataset utilized (7), the transcript or probe ID of CRP in each dataset $(7,10)$, the p-value of global differential expression, the F statistic for three-way comparisons, and the t statistic and $\mathrm{B}$ (log-odds of differential expression between the two groups compared) is displayed in this chart, provided by GEO2R. 
A

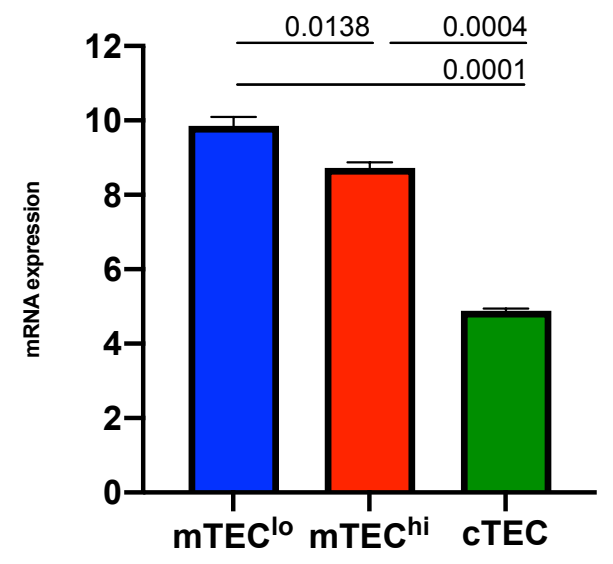

C

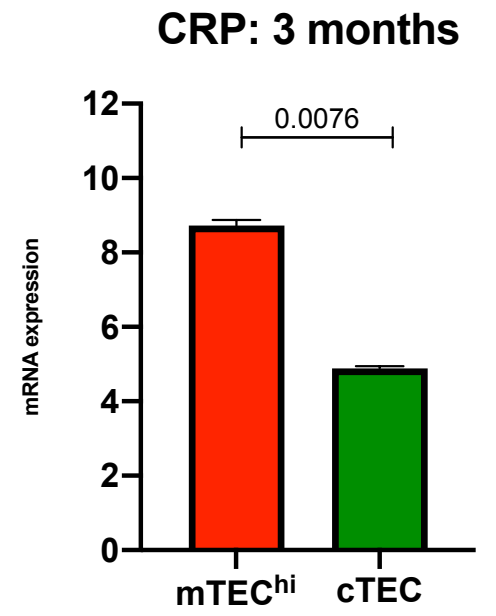

B

CRP: 3 months

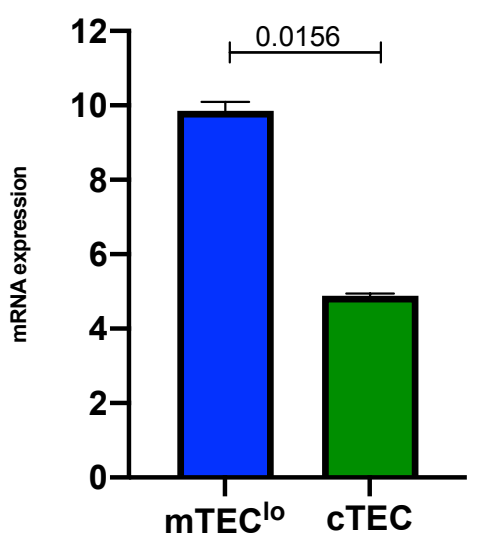

D

CRP: 3 months

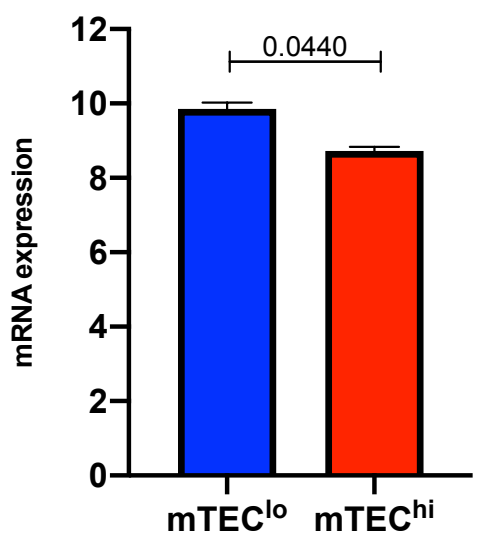

Figure 1: At 3 months, CRP is one of the most differentially expressed genes in the TEC transcriptome when comparing the global gene expression profiles of mTEClo, mTEChi, and CTEC

The expression of CRP is graphically represented as a bar graph with the error bars denoting the standard error of the mean, between mTEClo, mTEChi, and cTEC ( $n=2$ for each cell type). A statistical test was performed to evaluate the significance of difference between each cell type compared; for three-way comparisons (1A), an ordinary one-way ANOVA was performed; for two-way comparisons (1B-D), a two-tailed unpaired t-test with Welch's correction was performed. The results of each statistical test is shown on each graph as a $p$-value. 

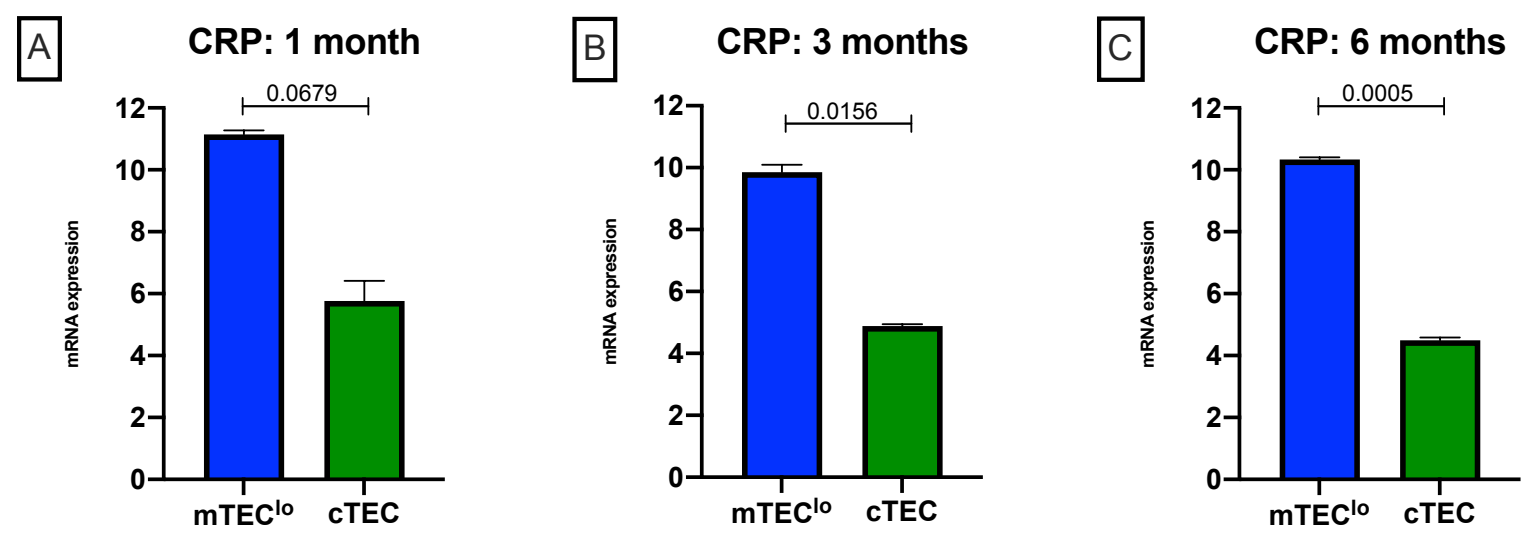

Figure 2: CRP is differentially expressed in a cell-type specific manner in the mTEClo of the thymus throughout murine development.

The expression of CRP is graphically represented as a bar graph with the error bars denoting the standard error of the mean, between mTEClo and cTEC ( $n=2$ for each cell type). A statistical test, a two-tailed unpaired t-test with Welch's correction was performed to evaluate the significance of difference between each cell type compared. The results of each statistical test is shown on each graph as a $p$-value. 